\title{
INITIAL EXPERIMENTAL STUDIES OF ELECTRON ACCUMULATION IN A HEAVY-ION BEAM*
}

\author{
A.W. Molvik ${ }^{1,2, *}$, D. Baca ${ }^{1,3}$, F.M. Bieniosek ${ }^{1,3}$, R.H. Cohen ${ }^{1,2,}{ }^{*}$, A. Friedman ${ }^{1,2}$, M.A. Furman ${ }^{3}$, E.P. \\ Lee $^{1,3}$, S.M. Lund ${ }^{1,2}$, L. Prost ${ }^{1,3}$, A. Sakumi ${ }^{4}$, P.A. Seidl ${ }^{1,3}$, J.-L. Vay ${ }^{1,3}$ \\ ${ }^{1}$ Virtual National Laboratory for Heavy Ion Fusion \\ ${ }^{2}$ Lawrence Livermore National Laboratory, Livermore, CA 94550 \\ ${ }^{3}$ Lawrence Berkeley National Laboratory, Berkeley, CA 94720 \\ ${ }^{4}$ RIKEN, (Now at CERN)
}

\begin{abstract}
Accelerators for heavy-ion inertial fusion energy (HIF) have an economic incentive to fit beam tubes tightly to beams, putting them at risk from electron clouds produced by emission of electrons and gas from walls. Theory and PIC simulations suggest that the electrons will be radially trapped in the $\geq 1 \mathrm{kV}$ ion-beam potential. We are beginning studies on the High-Current Experiment (HCX) with unique capabilities to characterize electron production and trapping, the effects on ion beams, and mitigation techniques. We are measuring the flux of electrons and gas evolved from a target, whose angle to the beam can be varied between $78^{\circ}$ and $88^{\circ}$ from normal incidence. Quadrupole magnets are operating with a variety of internal charged particle diagnostics to measure the beam halo loss, net charge, electron ionization rate, and gas density.
\end{abstract}

\section{INTRODUCTION}

Electron cloud effects (ECEs) are increasingly recognized as important, but incompletely understood, dynamical phenomena, which can severely limit the performance of colliders, the next generation of high-intensity rings, or future high-intensity heavy ion accelerators such as envisioned in Heavy Ion Inertial Fusion (HIF) [1].

Accelerators for HIF have an economic incentive to fit beam tubes tightly to beams. This places them at risk from gas desorption runaway, and from electron clouds produced by secondary electrons and ionization of gas. We have initiated an experimental and theoretical program to measure, understand, and model these effects in heavy-ion accelerators [2].

\section{HCX CAPABILITIES}

Theory and PIC simulations suggest that the electrons will be radially trapped in the $\geq 1 \mathrm{kV}$ ion-beam potential $[2,3]$, and can be detrapped by drifting into an upstream acceleration gap [2]. We have installed four quadrupole magnets on the High-Current Experiment (HCX) [4] at LBNL with internal electron diagnostics.

On HCX we are studying the transport of a $1 \mathrm{MeV}, 180$ $\mathrm{mA}, \mathrm{K}^{+}$ion beam. (HCX has also operated at $1.8 \mathrm{MeV}$, $500 \mathrm{~mA}$.) It has a beam potential of $\sim 1.5 \mathrm{kV}$, rise and fall times of $1 \mu \mathrm{s}$, and a flattop duration of $4 \mu \mathrm{s}$, repeated at $10 \mathrm{~s}$ intervals. Electron transit times between walls are in the range of $7 \mathrm{~ns}$, almost 3 orders of magnitude shorter *molvik1@1lnl.gov than the flattop duration. This enables exploration of unique electron trapping regimes: multipactor trapping will not occur during the flattop, however secondary electrons will be trapped during the rise time. Ionization of gas by the beam generates deeply trapped electrons, the ions from gas are expelled in $\leq 1 \mu \mathrm{s}$.

Trailing edge multipacting, if it occurs, will be at the end of the fall time when the bounce time of electrons between walls grows to $\geq 25 \mathrm{~ns}$ as the beam potential falls below $100 \mathrm{~V}$. Then electrons gain $\geq 40 \mathrm{eV}$ on each transit. But, all electrons should be lost before the next pulse, $10 \mathrm{~s}$ later in HCX, $0.2 \mathrm{~s}$ later in a future power plant driver. On PSR, electrons are observable for surprisingly long times, but still only until $1 \mu \mathrm{s}$ after a pulse [5]

HCX provides an opportunity to search for subtle electron trapping mechanisms. To elaborate - an electron emitted from the beam tube with a few $\mathrm{eV}$ is accelerated by the beam potential to $\sim 10^{3}$ higher energy, then decelerates towards the opposite wall. An irreversible conversion of only $\sim 10^{-3}$ of the peak radial energy to axial or azimuthal energy will trap the electron, preventing it from reaching the opposite wall.

We have used the Gas-Electron Source Diagnostic (GESD) on the HCX to measure the flux of electrons and gas evolved from a target, whose angle to the beam can be varied between $78^{\circ}$ and $88^{\circ}$ from normal incidence. The results will be discussed in subsequent sections.

We have installed a variety of charged particle diagnostics in quadrupole magnets to characterize electron production and trapping: (1) Electrodes, flush with the beam tube wall, are to measure the beam halo loss plus the resulting secondary electron emission. Using the electronemission coefficient measured with the GESD, we can infer the beam-halo loss. (2) Capacitive probes measure the net beam charge from which we can infer electron densities if they exceed a few percent of the beam density. (3) Grids shield collectors from the 3-orders-of-magnitude larger capacitive signal, to enable measurement of the current of expelled ions from ionization of gas. This ion current will be calibrated against an ion gauge, varying the pressure by leaking in a known gas. Then, we can determine the time dependence of gas density in the beam. It also directly measures the production rate of electrons from gas (when corrected by the ratio of the ionization cross section to the sum of ionization and charge-exchange cross sections). Slit scanners and beam profile diagnostics 
before and after the quadrupole magnets allow effects of electrons on the ion beam to be determined.

At present, the diagnostic bias power supplies, signal preamps, and data acquisition recorders and software produce reasonable signals. We are ready to commission the diagnostics to establish the validity of the information. Then we can proceed to experiments that study the two major sources of electrons: secondary emission from the beam tube and ionization of background and desorbed gas.

\section{ION SCATTERING FROM SURFACE}

We study ion scattering from surfaces with the TRIM (now SRIM) Monte Carlo code [6]. We found that large fractions of the incident beam ions "backscatter" near grazing incidence. Two consequences are:

(1) Halo ions in quadrupole magnets tend to be lost where the magnetic field is tangent to the beam tube, which confines secondary electrons to remain harmlessly near the wall [2]. But ion scattering allows energetic ions to impinge the beam tube at other azimuths where the field lines pass through the beam. Secondaries generated there could be trapped within the beam.

(2) Gridded collectors depend upon suppressing seconddary electrons, generated at one electrode, from reaching another. The initial gridded collectors are located only at azimuths where the magnetic field is tangent to the electrode surfaces, resulting in magnetic insulation of collectors from grids. These can measure expelled ions, but not currents of detrapped electrons.

\section{ELECTRON EMISSION}

On HCX, we use the GESD to measure electron emission and gas desorption from $1 \mathrm{MeV} \mathrm{K}^{+}$ions incident on a stainless-steel target near grazing incidence (Fig. 2). These data allow us to calibrate electrodes in magnetic quadrupoles that are flush with the beam tube: by measuring the secondary emission current, we infer the beam loss and the gas desorption. We also anticipate using the GESD to study mitigation techniques for reducing electron emission and gas desorption.

The surface of the target has been ground, with grooves parallel to the beam direction to minimize the ploughedfield variations in ion angle of incidence on a microscopic scale. Each electrode can be biased independently. The electron suppressor ring is biased to $-200 \mathrm{~V}$ to block electrons from entering or leaving the GESD.

To measure the beam current into the GESD, we raise the target to the upper position in Fig. 2, exposing a Faraday cup to the beam. We bias the grid to $-150 \mathrm{~V}$, and the target/Faraday cup to $-40 \mathrm{~V}$. The HCX beam is expanding over the $1.5 \mathrm{~m}$ drift to the GESD, so that the current into the GESD is $\sim 0.14 \mathrm{~mA}$, out of a total beam current of $180 \mathrm{~mA}$ at $1 \mathrm{MeV}$. Maintaining the ion-beam current at $<<1 \mathrm{~mA}$ is necessary to avoid limiting the electron emission current by space charge (the target to grid gap is $\sim 7 \mathrm{~cm}$ ) rather than by electron emission.

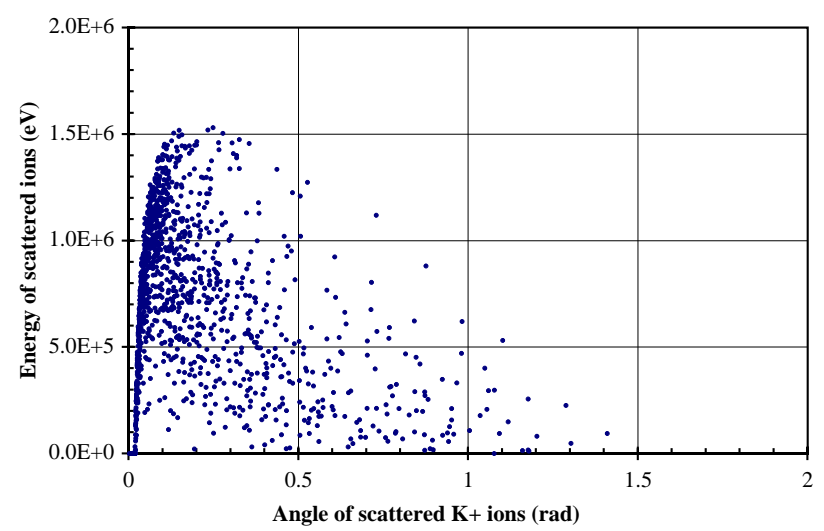

Figure 1. TRIM-98 results for $20001.8 \mathrm{MeV} \mathrm{K}^{+}$ions incident on stainless steel, $88^{\circ}$ from normal. The energy is shown Vs. the angle for the backscattered $64 \%$ of ions.

To measure the electron emission current, we bias the grid to $+150 \mathrm{~V}$ and the reflected ion catcher to $-25 \mathrm{~V}$, leaving the target at $-40 \mathrm{~V}$. The catcher is designed to "catch" about $90 \%$ of ions reflected (back-scattered) from the target. The SRIM code [6] calculates that $60-70 \%$ of ions incident on the target near grazing incidence will be reflected. We minimize the catcher current by biasing it $15 \mathrm{~V}$ positive relative to the target.

The electron emission coefficient is shown in Fig. 3(a). We apply models based on electron energy input from ion beam $\mathrm{dE} / \mathrm{dx}$ in matter. These models predict the magnitude [7] and the angular dependence of the electron emission coefficient $\eta_{\mathrm{e}}[8], \eta_{\mathrm{e}} \propto \mathrm{d} / \cos (\theta)$, where $\mathrm{d} / \cos (\theta)$ is the ion path length through a thin $\mathrm{d} \approx 2 \mathrm{~nm}$ thick surface layer (where secondary electrons originate).

The electron emission falls below the $1 / \cos (\theta)$ curve beyond $86^{\circ}$ due to large-angle nuclear scattering of ions out of the $2 \mathrm{~nm}$ layer. [8]. We tested this model for saturation with the SRIM 2003 code [6] by varying the thickness $\mathrm{d}$ of a thin foil with $1 \mathrm{MeV} \mathrm{K}^{+}$incident on $88^{\circ}$, until the transmitted distance normalized to $d / \cos (\theta)$ was near 0.75 [we settled for 0.78 as shown in Fig. 3(a)], the

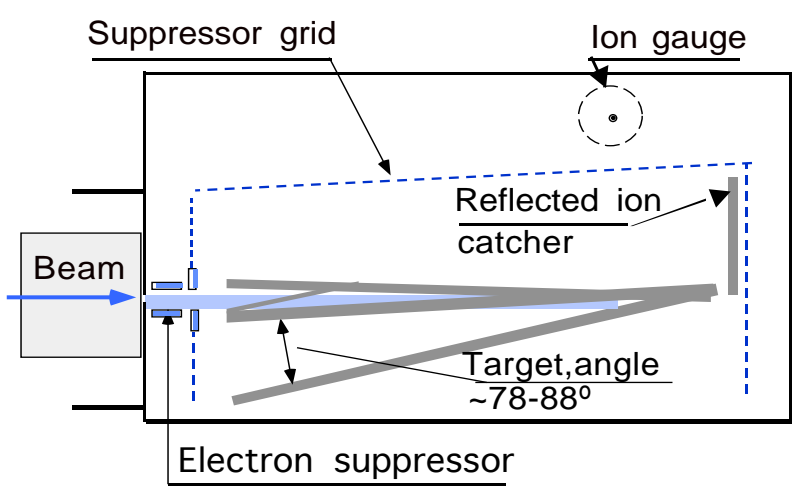

Figure 2. The Gas-Electron Source Diagnostic (GESD). Beam from the left is incident on a target whose angle of incidence can be varied between $78^{\circ}$ and $88^{\circ}$ relative to normal. The target is shown at 3 angles, the upper position centers a vee-shaped Faraday cup on the aperture. 

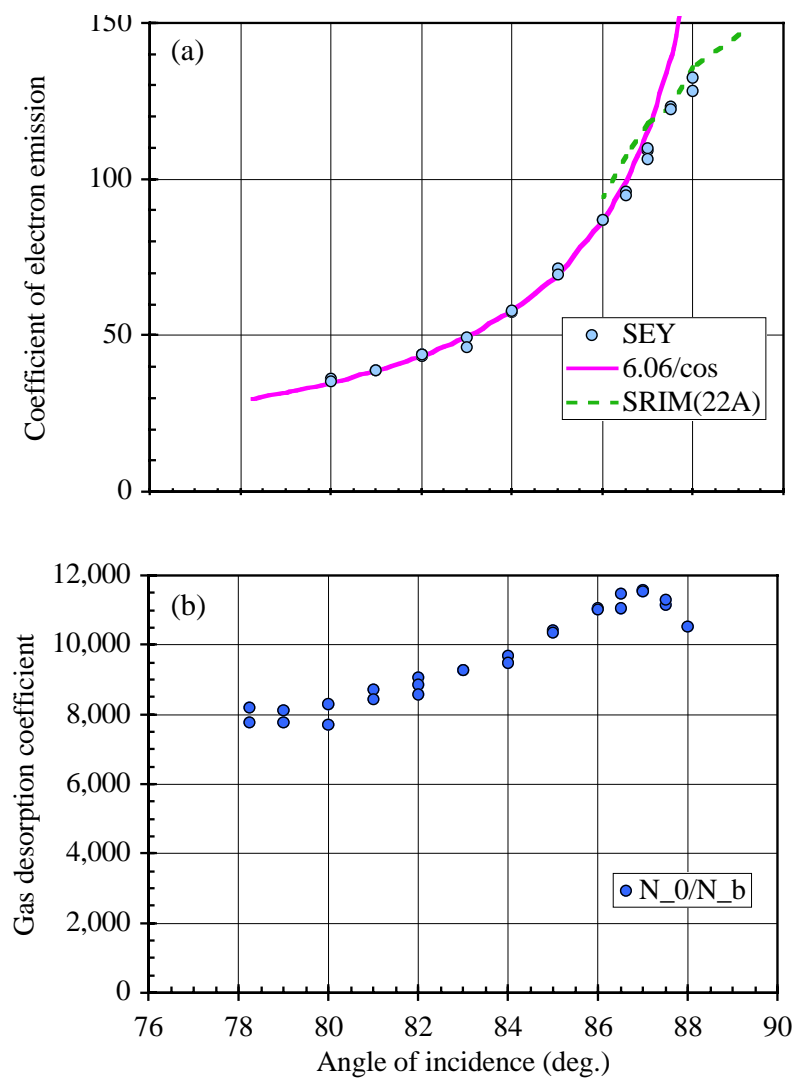

Figure 3. (a) Electron emission from $1 \mathrm{MeV} \mathrm{K}{ }^{+}$incident on stainless steel surface varies as $1 / \cos (\theta)$ with angle of incidence relative to normal. (b) Gas desorption varies more slowly with angle of incidence than $1 / \cos (\theta)$.

amount by which the measured $\eta_{\mathrm{e}}$ fell below $1 / \cos (\theta)$. We found that $d=2.2 \mathrm{~nm}$ satisfied this condition. Then using this thickness at other angles gave the dashed line curve in Fig. 3(a), which is a reasonable fit to the data at other angles near grazing incidence.

\section{GAS DESORPTION}

The gas desorption coefficient $\eta_{0}$, is measured from the pressure rise after a pulse. The GESD pumps out through the 0.3 by $2.5 \mathrm{~cm}$ entrance aperture, plus a $1 \mathrm{~cm}$ diameter hole, giving a pump-out time constant of $0.3 \mathrm{sec}$, long enough for an ion gauge to determine the peak pressure, but short compared with the $10 \mathrm{~s}$ before the next pulse. The less than $1 / \cos (\theta)$ dependence of gas desorption indicates that it is not only from layers of gas adsorbed on the surface (Fig. 2(b)).

We calibrated the GESD Bayard-Alpert ion gauge, over a range of emission currents from $0.5-10 \mathrm{~mA}$ and at two pressures 0.7 and $2.5 \times 10^{-6}$ Torr, against a Granville Phillips Stabil-Ion Gauge. The sensitivity varied with emission current over a range of $13 \%$, from a low of 7.7 Torr $^{-1}$ at $2 \mathrm{~mA}$ to about 8.7 Torr $^{-1}$ approaching $10 \mathrm{~mA}$. This compares with the nominal 10 Torr $^{-1}$ for the GESD gauge.

We compare two models for desorption: physical and electronic sputtering. Electronic sputtering is due to the electronic component of $\mathrm{dE} / \mathrm{dx}[9,10]$, which is evaluated with the SRIM code [6]. Physical sputtering results from the nuclear scattering component of $\mathrm{dE} / \mathrm{dx}$, but is much less than the electronic component for $\mathrm{K}^{+}$ions with energy exceeding $250 \mathrm{keV}$.

The major difficulty with the electronic sputtering model for desorption from accelerator beam tubes is that it is not expected to be applicable to ions impinging on metals, because free electrons rapidly neutralize charge separation in the ion track, preventing a coulomb explosion. Ion gauges have an analogous difficulty, they can only measure volatile molecules that can impinge walls many times without sticking. Measurements at CERN found the dominant desorbed gases to be $\mathrm{CO}, \mathrm{CO}_{2}, \mathrm{H}_{2}$, and $\mathrm{CH}_{4}$ [11], all insulators in solid or liquid form.

The compelling motivation to use this model is that much more energy is available from electronic stopping than from nuclear stopping in the energy range for heavyion fusion, and even more so for high-energy physics. With physical sputtering from the nuclear scattering, it is difficult to get coefficients greater than a few tens, but electronic sputtering is energetically capable of producing desorption coefficients in the range of thousands as observed here and at CERN [11].

\section{ACKNOWLEDGEMENT}

We are grateful to R. Hipple, W. Strelo and their staff for excellent technical support. This work was performed for the U.S. Department of Energy under contracts W7405-ENG-48 at U.C. Lawrence Livermore National Laboratory and DE-AC03-76F00098 at U.C. Lawrence Berkeley National Laboratory.

\section{REFERENCES}

[1] http://wwwslap.cern.ch/collective/ecloud02/ proceedings/index.html

[2] R. H. Cohen, et al., "Stray electron accumulation and effects in HIF accelerators", paper TOAA010 this conference.

[3] W.B. Herrmannsfeldt, Nuclear Instrum and Meth. in Phys. Res. A 464, 305 (2001).

[4] P.A. Seidl, D. Baca, F.M. Bieniosek et al., "The High Current Transport Experiment for Heavy Ion Fusion", paper ROAC001, this meeting (2003).

[5] R.J. Macek, et al., "Observation of an Intense Electron Cloud at the LANSCE Proton Storage Ring," LANSCE Div. Tech. Rev. (2002).

[6] J.F. Ziegler, http://www.srim.org/.

[7] P. H. Stoltz, M. A. Furman, J.-L. Vay, A.W. Molvik and R.H. Cohen, "Numerical simulation of the generation of secondary electrons in the High Current Experiment", Phys. Rev. ST Accel. Beams 6, 054701 (2003).

[8] P. Thieberger, et al., Phys. Rev. A61, 042901 (2000).

[9] W. L. Brown, et al., Phys. Rev. Lett. 45, 1632 (1980).

[10] W.L. Brown, R. E. Johnson, Nucl. Instrum. and Meth. B13, 295 (1986).

[11] E. Mahner, et al., Phys. Rev. Special Topics Accelerators and Beams 6, 013201 (2003). 